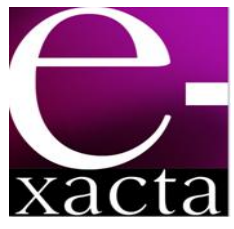

ISSN: 1984-3151

\title{
Óleo essencial de Satureja montana L.: ANÁlise CROMATOGRÁFICA, DETERMINAÇÃO DAS ATIVIDADES ANTIOXIDANTE E ANTIFÚNGICA
}

\author{
ESSENTIAL OIL OF THE SATUREJA MONTANA L .: CHROMATOGRAPHIC \\ ANALYSIS, DETEMINATION ANTIOXIDANT AND ANTIFUNGAL ACTIVITIES
}

\section{Lucilene Fernandes Silva ${ }^{1}$, Maria das Graças Cardoso ${ }^{2 *}$, Paulo Estevão de Souza ${ }^{3}$, Milene Aparecida Andrade ${ }^{4}$, Christiane Maria de Oliveira ${ }^{5}$, Marcos de Sousa Gomes ${ }^{6}$ Maria Luisa Teixeira ${ }^{7}$, Juliana de Andrade Santiago ${ }^{8}$}

1 Mestre em Agroquímica. DQI/UFLA, 2014. Doutoranda da Universidade Federal de Lavras - UFLA. Lavras, MG. lucilenesilva@dqi.ufla.br.

2 Doutora em Química. DQI/UFMG, 1995. Professora Titular do Departamento de Química da Universidade Federal de Lavras UFLA. Lavras, MG. mcardoso@dqi.ufla.br.

3 Doutor em Fitopatologia. DFP/UFLA, 1996. Professor Titular do Departamento de Fitopatologia da Universidade Federal de Lavras - UFLA. Lavras, MG. pauleste@dfp.ufla.br.

4 Doutora em Agroquímica. DQI/UFLA, 2013. Universidade Federal de Lavras - UFLA. Lavras, MG. mileneaandrade@hotmail.com

5 Mestre em Agroquímica. DQI/UFLA, 2015. Universidade Federal de Lavras - UFLA. Lavras, MG. chris.m.oliveira@hotmail.com.

6 Doutor em Agroquímica. DQI/ UFLA, 2014. Professor Associado do Departamento de Química na Universidade Federal dos Vales do Jequitinhonha e Mucuri-UFVJM. Unaí, MG. marcosopq@yahoo.com.br.

7 Mestre em Agroquímica. DQI/UFLA, 2013. Doutoranda da Universidade Federal de Lavras - UFLA. Lavras, MG. teixeira ml@hotmail.com.

8 Mestre em Agroquímica. DQI/UFLA, 2013. Doutoranda da Universidade Federal de Lavras - UFLA. Lavras, MG. juandrade quimica@yahoo.com.br.

Recebido em: 19/06/2015 - Aprovado em: 20/11/2015 - Disponibilizado em: 30/11/2015

RESUMO: As plantas constituem fontes importantes de compostos biologicamente ativos, muitos dos quais são protótipos de síntese de um grande número de fármacos. Muitos trabalhos científicos relatam que os óleos essenciais possuem propriedades antifúngicas, antibacterianas, inseticidas, antioxidante, entre outras. Essas atividades podem ser atribuídas aos constituintes presentes nos óleos essenciais, que desempenham funções de proteção aos organismos vivos, resguardando-os contra micro-organismos, insetos e produção excessiva de radicais livres. No presente estudo, objetivou-se caracterizar e quantificar os constituintes químicos do óleo essencial de Satureja montana L., avaliar as atividades antioxidantes pelos métodos do sequestro do radical $D P P H$ e $\beta$-caroteno/ácido linoleico e o efeito fungicida sobre os fitopatógenos Colletotrichum truncatum, Fusarium graminearum e Drechslera oryzae, pelo método de fumigação. Os constituintes majoritários encontrados no óleo 
essencial foram o timol, o carvacrol, o p-cimeno e o linalol. A atividade antioxidante do óleo essencial apresentou maior destaque diante do sistema $\beta$-caroteno/ácido linoleico. $O$ óleo essencial apresentou atividade fungicida para todos os fungos testados, sendo que a partir da concentração de $250 \mu \mathrm{gmL}^{-1}$ ocorreu a inibição total para todos os fitopatógenos em estudo.

PALAVRAS-CHAVE: Planta medicinal, Inibição oxidativa, Atividade fungicida.

ABSTRACT: Plants are important sources of biologically active compounds, many of which are prototypes for the synthesis of a large number of drugs. Many scientific studies report that essential oils have antifungal, antibacterial, insecticide, and antioxidant activities, among others. These activities can be attributed to the constituents present in the essential oils that play protective roles in living organisms, protecting them against micro-organisms, insects and excessive production of free radicals. The present study sought to characterize and quantify the chemical constituents of the essential oil of Satureja montana $L$. and to evaluate the antioxidant activity by the sequestration of the DPPH radicals and the $\beta$-carotene/linoleic acid method. The fungicidal effect against the plant pathogens Colletotrichum truncatum, Fusarium graminearum and Drechslera oryzae were evaluated by the fumigation method. The major constituents found in the essential oil were thymol, carvacrol, p-cymene and linalool. The essential oil antioxidant activity showed greater emphasis on the $\beta$-carotene system/linoleic acid. The essential oil has fungicidal activity to all fungi tested and as from the concentration of $250 \mu \mathrm{gmL}^{-1}$ there is complete inhibition for all pathogens in the study.

KEYWORDS: Medicanal plant, Oxidative inhibition, Fungicida activity.

\section{INTRODUÇÃO}

O gênero Satureja compreende plantas anuais ou perenes, semiarbustivas e aromáticas, apresentando folhas pequenas e arredondadas, que habitam em regiões áridas, ensolaradas e rochosas. Muitos membros deste gênero são conhecidos por seu caráter aromático e medicinal (CAVAR et al., 2008). A Satureja montana L. (Segurelha), pertencente à família Lamiaceae, é conhecida como segurelha de inverno ou segurelha montanhesa. A segurelha cresce melhor com temperaturas na faixa de $7^{\circ} \mathrm{C}$ a $26^{\circ} \mathrm{C}$. Essas plantas necessitam de alta luminosidade e 0 solo para o plantio dessa espécie deve ser drenado e moderadamente fértil. Embora possam crescer mesmo em solos pobres, estes devem apresentar umidade moderada quando as plantas são jovens. Quando estão bem desenvolvidas, pode-se reduzir a irrigação do solo (SLAVKOVSKA et al., 2001).

A utilização de óleos essenciais no controle fitossanitário tem sido comumente relatada. Pesquisas realizadas por Silva et al. (2015) evidenciam que a maior parte dos trabalhos referentes aos óleos essenciais descritos na literatura são relativos às suas atividades biológicas. A busca por novos métodos para o controle de pragas tem constituído um desafio para o homem, pois o uso intensivo e indiscriminado de substâncias sintéticas favoreceu a origem de pragas resistentes, além de causar efeitos negativos ao meio ambiente e aos organismos que possam vir a se expor a essas substâncias, devido à sua alta toxicidade.

Em decorrência dos efeitos adversos provocados pelos produtos comercializados, buscam-se alternativas naturais menos agressivas e dentre estas podem-se destacar os estudos realizados com óleos essenciais.

Os óleos essenciais também vêm sendo largamente estudados quanto às suas atividades antioxidantes, pois muitos compostos sintéticos empregados nas indústrias, com propriedades antioxidantes, podem provocar danos à saúde, entre os quais se destacam os efeitos carcinogênicos, a hiperplasia gastrointestinal e a redução de hemoglobina (ANDRADE et al., 2013). Devido a isso, atualmente tem ocorrido um aumento nas pesquisas científicas com o objetivo de encontrar substâncias naturais com atividade antioxidante similar à do composto sintético 
e dentre essas substâncias destaca-se os óleo essenciais.

Diante do exposto, o presente estudo teve como objetivo a avaliação da composição química e as atividades antioxidante e biológica do óleo essencial de Satureja montana L.

\section{Material e MÉtodos}

\subsection{Material Vegetal}

As folhas de Satureja montana L. foram obtidas no Mercado Municipal de Belo Horizonte - MG/Brasil, no mês de abril de 2011 .

\subsection{EXTRAÇÃo dO ÓLEO ESSENCIAL}

O óleo essencial de Segurelha (300 g de plantas) foi extraído por hidrodestilação, utilizando um aparelho de Clevenger modificado adaptado a um balão de fundo redondo com capacidade de 6 litros, por 2 h. 0 hidrolato obtido foi centrifugado em centrífuga de cruzeta horizontal (Fanem Baby I Modelo 206 BL), a 965,36 x G por dez minutos. O óleo essencial foi coletado com o auxílio de uma pipeta de Pasteur, acondicionado em frasco de vidro âmbar e levado à pistola de secagem. Após esses procedimentos, envolveu-se o frasco com o óleo essencial em papel alumínio e deixado sob refrigeração (ANVISA, 2010). Paralelamente às extrações, realizou-se o teste de umidade, seguindo a metodologia descrita por Pimentel et al. (2006).

\subsection{ANÁLISE QUALITATIVA E QUANTITATIVA DOS COMPONENTES DO ÓLEO ESSENCIAL}

A análise qualitativa e quantitativa do óleo essencial foi realizada por cromatografia em fase gasosa, acoplada à espectrometria de massas (CG/EM Shimadzu, modelo QP 5050A), sob as seguintes condições experimentais: coluna capilar de sílica fundida (30m x 0,25mm) com fase ligada DB5 $(0,25 \mu \mathrm{m}$ de espessura de filme); He como gás de arraste com fluxo de $1,0 \mathrm{mLmin}^{-1}$. A temperatura foi programada, mantendo-se $50^{\circ} \mathrm{C}$ por $1,5 \mathrm{~min}$, seguido de um aumento de $4{ }^{\circ} \mathrm{Cmin}^{-1}$ até atingir $200{ }^{\circ} \mathrm{C}$, depois a $10^{\circ} \mathrm{C}$, até atingir $250^{\circ} \mathrm{C}$, mantendo-se constante essa temperatura por $5 \mathrm{~min}$. A temperatura do injetor foi de $250 \stackrel{\circ}{ } \mathrm{C}$ e a temperatura do detector (ou interface), de $280 \stackrel{\circ}{0}$. O volume da amostra injetada foi de $0,5 \mu \mathrm{L}$ em acetato de etila. A taxa de partição do volume injetado foi de 1:100 e pressão na coluna, de 64.20 $\mathrm{kPa}$.

As condições do espectrômetro de massas foram: detector de varredura 1.000, com intervalo de varredura de 0,50 fragmento e fragmentos detectados na faixa de 40 a 500 Da. A identificação dos constituintes foi realizada com base na comparação dos índices de retenção da literatura (ADAMS, 2007). Para o índice de retenção, foi utilizada a equação de Van den Dool e Kratz 1963 em relação à série homóloga de alcanos lineares $\left(\mathrm{nC}_{8}-\mathrm{nC}_{18}\right)$, do octano ao octadecano, fazendo extrapolação para $\mathrm{C}_{19}$ e $\mathrm{C}_{20}$ (nonadecano ao dodecano). Também foram utilizadas duas bibliotecas comerciais NIST107 e NIST21, que permitem a comparação dos dados dos espectros com aqueles existentes na biblioteca.

\subsection{ANÁLISE DA ATIVIDADE ANTIOXIDANTE}

\subsubsection{MÉtodo de SEQUestro de RAdicais DPPH}

A avaliação da atividade antioxidante pelo método de sequestro de radicais DPPH foi realizada de acordo com as metodologias de Sousa et al. (2007) e LopesLutz et al. (2008), seguidas de pequenas modificações. Em balões volumétricos, prepararam-se as soluções para utilização no ensaio, DPPH em metanol na concentração de $40 \mu \mathrm{g} \mathrm{mL}^{-1}$ (mantida sob 
refrigeração e ao abrigo da luz) e óleo essencial nas concentrações de 50,100,125, 250, 500, 750 e 1000 $\mu \mathrm{gmL}^{-1} \mathrm{em}$ metanol. Para o branco foram adicionados 0,3 mL da solução de maior concentração do óleo essencial e 2,7 mL da solução de DPPH. Para o controle foram empregados $0,3 \mathrm{~mL}$ de metanol e 2,7 $\mathrm{mL}$ da solução de DPPH e para a análise do óleo essencial foram adicionados $0,3 \mathrm{~mL}$ de solução de cada amostra e 2,7 mL da solução de DPPH. As leituras de absorbância foram realizadas em espectrofotômetro Shimadizu UV-1601PC a 517 nm, no tempo de 60 minutos após a mistura das duas soluções.

A atividade antioxidante foi determinada a partir da equação matemática \%AA $=100-[($ As $\times 100) / A c]$, sendo $A s=$ absorbância da amostra e $A c=$ absorbância do controle.

\subsubsection{MÉTODO B-CAROTENO/ÁCIDO LINOLEICO}

A determinação da atividade antioxidante foi realizada pelo método $\beta$-caroteno/ácido linoleico de acordo com a metodologia proposta por Lopes-Lutz et al. (2008).

Foi preparada uma emulsão de $\beta$-caroteno/ácido linoleico pesando-se $60 \mathrm{mg}$ de ácido linoleico, $6 \mathrm{mg}$ de $\beta$-caroteno e $600 \mathrm{mg}$ de Tween 20, adicionados em um balão de fundo redondo com $30 \mathrm{~mL}$ de clorofórmio. Removeu-se o clorofórmio em um evaporador rotatório e adicionaram-se $150 \mathrm{~mL}$ de água saturada de oxigênio. Repetiu-se o mesmo procedimento sem a adição do $\beta$-caroteno para 0 branco. Foram preparadas soluções do óleo essencial nas concentrações de 50,100, 125, 250, 500, 750 e 1000 $\mu \mathrm{g} \mathrm{mL}^{-1}$ em metanol.

As misturas reacionais foram preparadas em tubos de ensaio, em que $0,2 \mathrm{~mL}$ do óleo essencial foram adicionados juntamente com $2,5 \mathrm{~mL}$ de emulsão do $\beta$ caroteno. Para o branco foram adicionadas $2,5 \mathrm{~mL}$ de emulsão sem adição do $\beta$-caroteno e $0,2 \mathrm{~mL}$ da solução de maior concentração do óleo essencial. Para o controle foram acrescidos $0,2 \mathrm{~mL}$ de metanol e $2,5 \mathrm{~mL}$ de emulsão com $\beta$-caroteno. Foram realizadas leituras no comprimento de onda de $470 \mathrm{~nm}$ no tempo inicial e após 60 minutos de reação, onde as amostras foram incubadas a $50 \stackrel{\circ}{\circ}$.

A determinação da atividade antioxidante foi feita por meio da expressão matemática \%AA $=100$ [1-(AiAt)/Ac-Act], sendo $A i=$ absorbância da amostra no tempo zero; $A t=$ absorbância da amostra após $60 \mathrm{~min}$; $A c=$ absorbância do controle no tempo zero e Act= absorbância do controle após 60 minutos.

\subsection{ENSAIOS BIOLÓGICOS}

A avaliação do efeito fungicida do óleo essencial de Satureja montana L. foi realizada no Laboratório de Epidemiologia e Manejo do Departamento de Fitopatologia da Universidade Federal de Lavras.

Os fitopatógenos foram obtidos da coleção micológica do Laboratório de Patologia de Sementes- LAPS, registrados com os seguintes números: Colletotrichum truncatum LAPS-432; Fusarium graminearum LAPS431 e Drechslera oryzae LAPS-430.

Empregou-se o método de fumigação in vitro em meio de cultura BDA (batata, dextrose e ágar), avaliando-se o efeito do óleo essencial de Satureja montana L. sobre o crescimento e/ou a inibição micelial dos fitopatógenos (GOMES et al., 2013).

Nos ensaios foram utilizadas placas de Petri de $9 \mathrm{~cm}$ de diâmetro e $1,5 \mathrm{~cm}$ de altura, sendo adicionados 25 $\mathrm{mL}$ de meio de cultura em cada placa. Para a preparação dos tratamentos, o óleo essencial foi diluído em 0,25 mL de éter etílico, obtendo-se as seguintes concentrações: 50, 100, 125, 250, 500, 750 e $1000 \mathrm{\mu gmL}^{-1}$. Essas soluções foram aplicadas em cada placa sobre um papel de filtro circular de $4,00 \mathrm{~cm}$ de diâmetro, aderido à parte superior da placa de 
Petri. Posteriormente à solidificação do meio de cultura, discos de $7 \mathrm{~mm}$ de diâmetro foram cortados de uma cultura pura e adicionados no centro das placas. As placas foram vedadas com filme plástico e levadas para a câmara de crescimento, com temperatura de aproximadamente $25^{\circ} \mathrm{C}$ e fotoperíodo de 12 h. Paralelamente, foram preparadas duas testemunhas: uma composta apenas do fitopatógeno e do meio de cultura (testemunha absoluta) e outra testemunha relativa, onde foram colocados $0,25 \mathrm{~mL}$ de éter etílico no disco e o fitopatógeno.

As medidas do crescimento micelial das culturas dos respectivos fungos foram realizadas no décimo segundo dia após a inoculação do fitopatógeno Colletotrichum truncatum e no oitavo dia para o Fusarium graminearum e o Drechslera oryzae. Foram traçadas duas retas perpendiculares pela placa de Petri, passando pelo centro do disco de $9 \mathrm{~mm}$, e as leituras foram realizadas pelas medidas do diâmetro de crescimento das colônias. A porcentagem de inibição do crescimento micelial foi calculada para cada dosagem em relação à testemunha absoluta.

\subsection{Delineamento estatístico}

O delineamento experimental utilizado foi 0 inteiramente casualizado, com três repetições. Os dados foram submetidos à análise de variância e as médias obtidas foram comparadas pelo Teste de Tukey, ao nível de $5 \%$ de probabilidade, utilizando o programa estatístico SISVAR (Ferreira, 2011).

\section{Resultados e Discussão}

O rendimento médio do óleo essencial de Satureja montana L. foi de $0,59 \%(\mathrm{~m} / \mathrm{m}$ Base Livre de Umidade(BLU)). Cavar et al. (2008) obtiveram para a mesma planta o rendimento de $0,5 \%$, corroborando o resultado encontrado neste trabalho.
Avaliando a constituição química do óleo essencial, pôde-se identificar 24 componentes, totalizando $97,45 \%$ da amostra. Observa-se pela TAB. 1 que os constituintes majoritários foram o timol (32,85\%), carvacrol $(14,35 \%)$, o $p$-cimeno $(12,12 \%)$ e o linalol (8,99\%). Trabalhos de Slavkovska et al. (2001), encontraram como constituintes majoritários do óleo essencial de Satureja montana L., o linalol (33\%), o $p$ cimeno $(43 \%)$, o borneol (10\%) e o trans-sabineno $(13 \%)$, resultados diferentes dos encontrados no presente estudo. Cavar et al. (2008), estudando diferentes espécies de Segurelha, coletadas em locais distintos na cidade de Trebinje (Bósnia e Herzegovina), encontraram proporções dos constituintes majoritários, variando de $28,6 \%$ a $31,7 \%$ para o timol e $23,3 \%$ a 27,9\% para o carvacrol. Marin et al. (2012), estudando o óleo essencial de Satureja montana L., encontraram como constituintes majoritários o carvacrol $(44,5 \%)$, seguido por $p$-cimeno (16,9\%), $\gamma$-terpineno $(8,7 \%)$, borneol $(5,1 \%)$ e timol (3,5\%). A composição química do óleo essencial de Segurelha desta região se diferencia dos constituintes relatados em outros trabalhos, principalmente para o constituinte timol.

A diferença na composição química dos óleos essenciais é influenciada por diversos fatores como o clima, o solo, a época da coleta, a idade e o desenvolvimento da planta, bem como dos diferentes órgãos vegetais, que também são de considerável importância e podem influenciar não só a quantidade total de metabólitos produzidos, mas também as proporções relativas dos componentes da mistura (GOBBO-NETO e LOPES, 2007). 
Tabela 1

Constituintes presentes no óleo essencial de Segurelha

\begin{tabular}{|c|c|c|c|}
\hline Pico & ${ }^{*} \mathrm{TR}$ (min) & Composto & (\%) CG-EM \\
\hline 1 & 7,525 & Tricicleno & 1,56 \\
\hline 2 & 8,065 & a-Pineno & 0,65 \\
\hline 3 & 9,044 & 1-Octen-3-ol & 1,48 \\
\hline 4 & 9,382 & Mirceno & 0,33 \\
\hline 5 & 10,370 & $\alpha$-Terpineno & 0,89 \\
\hline 6 & 10,650 & $p$-Cimeno & 12,12 \\
\hline 7 & 10,816 & Limoneno & 0,48 \\
\hline 8 & 10,936 & 1,8-Cineol & 1,09 \\
\hline 9 & 11,883 & $\mathrm{y}$-Terpineno & 1,89 \\
\hline 10 & 12,335 & $\mathrm{NI}$ & 1,24 \\
\hline 11 & 13,442 & Linalol & 8,99 \\
\hline 12 & 13,702 & cis-Tujona & 0,73 \\
\hline 13 & 15,227 & Cânfora & 1,27 \\
\hline 14 & 16,167 & Borneol & 5,06 \\
\hline 15 & 16,483 & Terpinen-4-ol & 4,59 \\
\hline 16 & 17,020 & $\alpha$-Terpineol & 1,91 \\
\hline 17 & 18,601 & Timol metil éter & 1,51 \\
\hline 18 & 19,024 & $\mathrm{NI}$ & 0,73 \\
\hline 19 & 20,481 & Timol & 32,85 \\
\hline 20 & 20,764 & Carvacrol & 14,35 \\
\hline 21 & 25,030 & $(E)$-Cariofileno & 2,89 \\
\hline 22 & 27,902 & $\beta$-Bisaboleno & 1,04 \\
\hline 23 & 30,092 & $\mathrm{NI}$ & 0,58 \\
\hline 24 & 30,276 & Óxido Cariofileno & 1,77 \\
\hline
\end{tabular}

${ }^{*} \mathrm{TR}=$ tempo de retenção

$\mathrm{NI}=$ não identificado

As porcentagens das atividades antioxidantes encontradas para o óleo essencial de Satureja montana L. diante dos dois métodos de análise estão descritas na FIG. 1. Pode-se observar que pelo método $\beta$-caroteno/ácido linoleico o óleo essencial apresentou maior atividade na concentração de 1000 $\mu \mathrm{gmL}^{-1}$. Os dois testes apresentaram respostas que não diferiram significativamente, contudo nas demais concentrações o óleo essencial teve maior atividade no método $\beta$-caroteno/ácido linoleico.

As estruturas dos constituintes majoritários timol, carvacrol, $p$-cimeno e linalol são importantes no papel de desempenhar atividade antioxidante, pois compostos contendo átomos de hidrogênio alílicos e/ou benzílicos mostram melhor atividade pela fácil abstração do hidrogênio atômico desses grupos funcionais, possibilitando assim uma atividade antioxidante significativa (LARSON, 1997).

O método $\beta$-caroteno/ácido linoleico é um método em que as condições são mais favoráveis para demonstrar a atividade antioxidante dos óleos essenciais, já que nesse ensaio os constituintes lipofílicos estão em contato com solução lipofílica, o que permite melhor solubilização do óleo essencial. Pelo método DPPH, o óleo essencial fica em contato com reagentes hidrofílicos, como o metanol, o que pode ser um fator importante para uma menor atividade antioxidante do óleo essencial neste ensaio (ANDRADE et al., 2013).

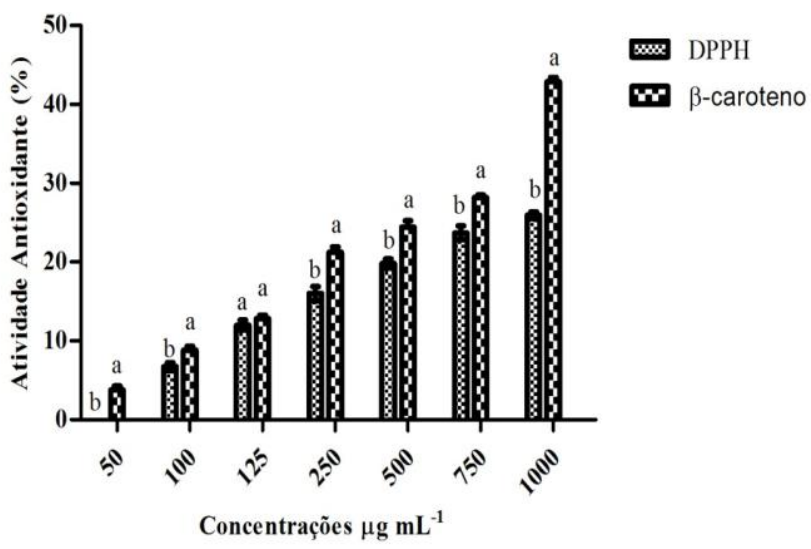

Figura 1 - Comparação da atividade antioxidante pelo método $\beta$-caroteno/ácido linoleico versus DPPH.

* Médias seguidas pela mesma letra em cada concentração, não diferem entre si pelo teste de Tukey a $5 \%$ de probabilidade.

O método $\beta$-caroteno/ácido linoleico prevê a capacidade do óleo em proteger membranas, enquanto o método DPPH avalia a atividade do óleo 
essencial em sequestrar radicais livres. Pelos resultados obtidos, pode-se propor que o óleo essencial apresenta maior capacidade em proteger membranas. Analisando as concentrações em que os testes foram efetuados, pode-se ressaltar que 0 aumento das concentrações possibilita um acréscimo nas atividades antioxidantes, demonstrando um efeito dose-dependente.

Cavar et al. (2008), utilizando o método DPPH, atribuíram a atividade antioxidante do óleo essencial das espécies Satureja montana e Satureja subspicata aos constituintes timol e carvacrol. Grosso et al. (2009), empregando o método do radical DPPH, mostraram que a combinação dos constituintes timol, carvacrol e timoquinona no óleo essencial de Segurelha são os responsáveis pela atividade antioxidante deste óleo. Milos e Makota (2011), estudando a atividade antioxidante do timol, $p$-cimeno, carvacrol e timoquinona, concluíram que a atividade antioxidante decresce na seguinte ordem: timol, carvacrol, timoquinona e p-cimeno, com os dois últimos apresentando atividades semelhantes. O timol e o carvacrol por serem isômeros de posição e com uma hidroxila ligada ao aromático apresentam atividade antioxidante melhor, sendo que nesse caso o timol apresenta hidroxila próxima a grupo alquila que pode, portanto, ser melhor estabilizado que 0 carvacrol. Já a timoquinona e o $p$-cimeno apresentam atividades semelhantes e inferiores ao timol e ao carvacrol por não apresentarem hidroxilas em suas estruturas, o que confere menor disponibilidade de doação de hidrogênios para os radicais formados, reduzindo assim a atividade antioxidante dessas estruturas.

O efeito do óleo essencial de Satureja montana L. sobre o crescimento micelial dos fitopatógenos Colletotrichum truncatum, Fusarium graminearum e Drechslera oryzae pode ser observado na FIG. 2.

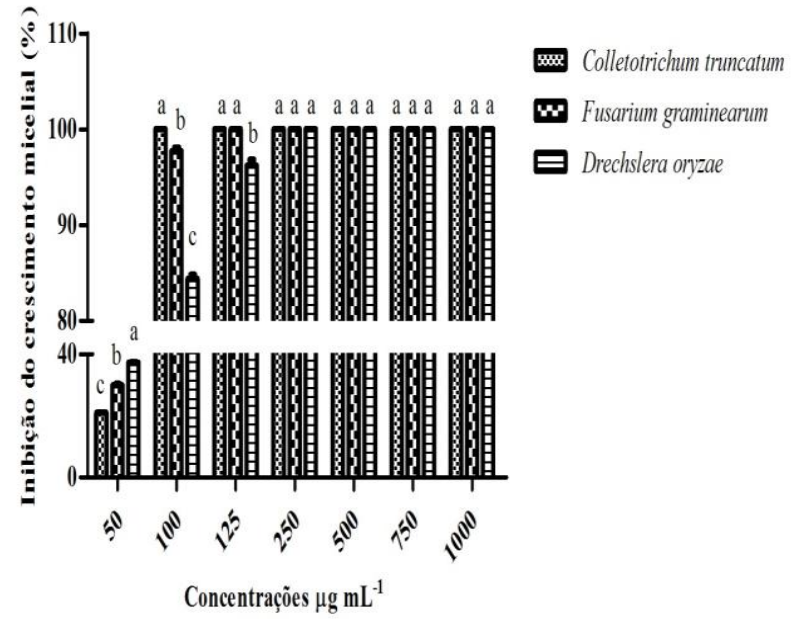

Figura 2 - Atividade antifúngica do óleo essencial de Satureja montana $L$.

* Médias seguidas pela mesma letra em cada concentração, não diferem entre si pelo teste de Tukey a $5 \%$ de probabilidade.

Foi observada para a testemunha absoluta (T.A.) e para a testemunha relativa (T.R.) a porcentagem de inibição de $0 \%$, não apresentando diferença estatística para os fungos testados. Portanto, pôde-se concluir que o solvente não interferiu na inibição dos fungos.

Nota-se pela FIG. 2 que na concentração de $50 \mu \mathrm{g}$ $\mathrm{mL}^{-1} \mathrm{o}$ óleo essencial apresentou maior eficiência em inibir o fungo Drechslera oryzae, seguido do Fusarium graminearum e menos eficiência para o Colletotrichum truncatum. Avaliando a concentração de $100 \mathrm{gg} \mathrm{mL}^{-1}$ a situação modifica-se e o óleo essencial apresenta inibição total do fitopatógeno Colletotrichum truncatum, seguido do Fusarium graminearum, tornando-se menos eficiente para o Drechslera oryzae. Na concentração de $125 \mu \mathrm{g} \mathrm{mL}^{-1}$, o óleo essencial não apresentou diferença significativa entre os fungos Colletotrichum truncatum e Fusarium graminearum, verificando-se a inibição total da colônias; e para o Drechslera oryzae observou-se uma inibição média de 96,19\%. Para as concentrações de 250, 500750 e $1000 \mu_{g m L^{-1}}$, o óleo essencial apresentou inibição total dos fitopatógenos em estudo.

Vieira et al. (2012), avaliando a atividade fungitóxica do óleo essencial de Ageratum conyzoides L. sobre os 
fitopatógenos Alternaria alternata, Colletotrichum gloesporioides, Fusarium oxysporum cubense, Bipolares sorokoniana e Rizoctonia solani, observaram efeito mais pronunciado sobre os dois últimos patógenos para os quais o óleo essencial apresentou $100 \%$ de inibição.

Velluti et al. (2004), realizando uma triagem inicial para a atividade inibitória de óleos essenciais para o crescimento de Fusarium verticillioides, Fusarium proliferatum e Fusarium graminearum, observaram que o óleo essencial de canela (Cinnamomum zeylanicum.) e o de cravo (Syzygium aromaticum) reduziram o crescimento de colônias de Fusarium graminearum em todas as condições testadas. $\mathrm{Na}$ concentração de $1000 \mathrm{mgmL}^{-1}$, os óleos de cravo e de canela reduziram o crescimento de colônias cerca de $62 \%$ e $80 \%$, respectivamente, sendo o óleo essencial de segurelha mais eficaz contra o fitopatógeno, pois na concentração de $250 \mu \mathrm{gmL}^{-1}$ obteve-se a inibição total.

Begum et al. (2007), estudando a composição e a atividade antifúngica do óleo essencial das folhas de Cinnamomum verum Presl. sobre o fungo Drechslera oryzae, comprovaram que o óleo essencial apresentou concentração mínima inibitória de $750 \mu \mathrm{gmL}^{-1}$, valores acima daqueles aqui encontrados, comprovando que 0 patógeno é mais sensível ao óleo essencial de segurelha. Entre todos os micro-organismos estudados, o fungo Drechslera oryzae foi o mais resistente, apresentando uma inibição total em uma concentração superior aos demais fungos estudados.

\section{CONCLUSÃo}

O óleo essencial de Segurelha montana L. apresentou rendimento de $0,59 \% \mathrm{p} / \mathrm{p}$. Os constituintes majoritários encontrados no óleo essencial foram o timol $(32,5 \%)$, o carvacrol (14,35\%), p-cimeno (12,2\%) e linalol $(8,9 \%)$.

A atividade antioxidante foi mais expressiva perante 0 método $\beta$-caroteno/ácido linoleico.

O óleo essencial apresentou atividade fungicida sobre Colletotrichum truncatum, Fusarium graminearum e Drechslera oryzae a partir da concentração de $250 \mu \mathrm{g}$ $\mathrm{mL}^{-1}$, pois apresentou $100 \%$ de inibição para todos os fitopatógenos em estudo, podendo assim ter uma gama de aplicações como na conservação de alimentos, utilização na cosmetologia para produção de produtos antienvelhecimento devido sua atividade antioxidante, assim como utilização na agricultura para diminuição de danos causados por micro-organismos como fungos que causam grandes perdas na colheita e também no pós colheita.

\section{AGRADECIMENTOS}

Ao Conselho Nacional de Desenvolvimento Científico e Tecnológico (CNPq), à Fundação de Amparo à Pesquisa do Estado de Minas Gerais (FAPEMIG) e à Coordenação de Aperfeiçoamento de Pessoal de Nível Superior (CAPES).

\section{REFERÊNCIAS}

ADAMS, R. P. Identification of Essential Oils Components by Gas chromatography/Mass Spectrometry, 4. ed. Carol Stream: Allured, 2007. 804p. ISBN 1932636219.
ANVISA. AGÊNCIA NACIONAL DE VIGILÂNCIA SANITÁRIA. Farmacopeia brasileira. 5. ed. Brasília, 2010. v. 1, p. 198-199. Disponível em:

<http://www.anvisa.gov.br/hotsite/cd_farmacopeia/inde x.htm>. Acesso em: 12 dez. 2013. 
ANDRADE, M. A. et al. Chemical Composition and Antioxidant Activity of Essential Oils from Cinnamodendron dinisii Schwacke and Siparuna guianensis Aublet. Antioxidants, v.2, n.1, 384-397, 2013. ISSN 2076-3921.

BEGUM, J. et al. Composition and antifungal activity of essential oil of leaves of Cinnamomum verum Presl. grown in Bangladesh. Indian Perfumer, Shakarpur, v.51, n.1, $15-18,2007$.

CAVAR, S. et al. Chemical composition and antioxidant and antimicrobial activity of two Satureja essential oils. Food Chemistry, Chicago, v.111, n.3, $648-653,2008$. ISSN 0308-8146.

FERREIRA, D. F. Sisvar: a computer statistical analysis system. Ciência e Agrotecnologia, Lavras, v.35, n.6, 1039 - 1042, 2011. ISSN 1981-1829.

GOBBO-NETO, L.; LOPES, N. P. Plantas medicinais: Fatores de influência no conteúdo de metabólitos secundários. Química Nova, São Paulo, v.30, n.2, 374 - 381, 2007. ISSN 0100-4042.

GOMES, M. S. et al. Multivariate analysis of the essential oil components of the genus Citrus and their antifungal activity. Científica, v.41, n.2, 111-121, 2013 ISSN: 1984-5529.

GROSSO, C. et al. Antioxidant activities of the supercritical and conventional Satureja montana extracts. Journal of Food Science, Pinerolo, v.74, n.1, 713 - 717, 2009. ISSN 1750-3841.

LARSON, R. A.. Naturally occurring antioxidants. Urbana Champ, Illinois, USA: CRC Press., 1997, p. 70.

LOPES-LUTZ, D. et al. Screening of chemical composition, antimicrobial and antioxidant activities of Artemisia essential oil. Phytochemistry, Egham, v.69, n.1, 1732 - 1738, 2008. ISSN 0031-9422.

MARIN, M. et al. Antioxidative, antibacterial and antifungal activity of the essential oil of wild-growing
Satureja montana L. from Dalmatia, Croatia. Flavour and Fragrance Journal, Chichester, v.27, 216 - 223, 2012. INSS 0000-3082.

MILOS, M.; MAKOTA, D. Investigation of antioxidant synergisms and antagonisms among thymol, carvacrol, thymoquinone and $p$-cymene in a model system using the Briggs-Rauscher oscillating reaction. Food Chemistry, Chicago, v.1016, n.10, 296 - 299, 2011. INSS 0308-8146.

PIMENTEL, F. A. et al. Convenient method for the determination of moisture in aromatic plants. Química Nova, São Paulo, v.29, n.2, 373 - 375, 2006. INSS 0100-4042.

SILVA, L. F. et al. Chemical characterization, antibacterial and antioxidant activities of essential oils of Mentha viridis $\mathrm{L}$. and Mentha pulegium $\mathrm{L}$. (L).

American Journal of Plant Sciences, v.6, n.1, 666675, 2015. INSS 2158-2750.

SLAVKOVSKA, V. et al. Variability of essential oils of Satureja montana L. and Satureja kitaibelii Wierzb. ex heu. from the central part of the balkan peninsula. Phytochemistry, Egham, v.57, n.1, 71 - 76, 2001. ISSN 0031-9422.

SOUSA, C. M. M. et al. Fenóis totais e atividade antioxidante de cinco plantas medicinais. Química Nova, São Paulo, v.30, n.2, 351 - 355, 2007. ISSN 0100-4042.

VELLUTI, A. et al.. Initial screening for inhibitory activity of essential oils on growth of Fusarium verticillioides, $F$. proliferatum and $F$. graminearum on maize-based agar media. Food Microbiology, Amsterdam, v.21, n.1, 649 - 656, 2004. ISSN 07400020.

VIEIRA, S. S. et al. Composição química e atividade fungitóxica do óleo essencial de Ageratum conyzoides L. (Mentrasto). Magistra, Cruz das Almas, v.24, n.1, 55 - 62, 2012. ISSN 2236-4420. 